\title{
Triangulation of Input-Output Tables Based on Mixed Integer Programs for Inter-temporal and Inter-regional Comparison of Production Structures
}

\section{Yasushi Kondo}

Received: 2 October 2013 / Accepted: 18 March 2014 / Published online: 15 April 2014 (C) 2014 Y. Kondo; licensee Springer. This is an Open Access article distributed under the terms of the Creative Commons Attribution License (http://creativecommons.org/licenses/by/2.0), which permits unrestricted use, distribution, and reproduction in any medium, provided the original work is properly cited.

\begin{abstract}
Understanding the industrial structure of a national or regional economy is one of the central issues in economics. The triangulation of an input-output table (IOT) can be employed to understand the production structure of an economy. Intertemporal and inter-regional comparisons of multiple IOTs have addressed interesting and important issues pertaining to international trade, economic growth, and interindustry relationships in the economy. Rank correlation coefficients between sector rankings obtained by solving optimization problems have been utilized to quantify similarities among production structures. However, it is well known that calculated rank correlations might be weak even if underlying structures are similar because the optimization problem inherently has multiple optimal solutions, thus leading to erroneous interpretations. This paper proposes a new method to triangulate IOTs based on mixed integer programs (MIPs) for comparing the production structures of multiple economies. The proposed new method does not suffer from non-uniqueness of optimal solutions and is consistent with maximization of the Kendall rank correlation coefficient. The application of the proposed method to the Japanese economy demonstrates stability of the Japanese production structure during 1995-2005. Comparisons of triangulated IOTs further reveal similarities in production structures of the Chinese, Japanese, and the U.S. economy for the year 2009.
\end{abstract}

Keywords Triangularization · Fundamental structure of production · Production structure $\cdot$ Mixed integer program $\cdot$ Input-output table

JEL Classification C61 - C67 · L16

Electronic supplementary material The online version of this article (doi:10.1186/2193-2409-3-2) contains supplementary material.

Y. Kondo (凶)

Faculty of Political Science and Economics, Waseda University, 1-6-1 Nishi-waseda, Shinjuku-ku, Tokyo, 169-8050 Japan

e-mail: ykondo@waseda.jp 


\section{List of Abbreviations}

IOT: Input-output table

MIP: $\quad$ Mixed integer program

IP: Integer program

WIOD: World Input-Output Database

\section{Introduction}

The entire production structure of a national or regional economy can be represented through its input-output table (IOT), consisting of extensive numerical data on different branches of national and regional economies. Quantitative methods for summarizing and visualizing information archived in IOTs are indispensable, since understanding the industrial structure of an economy is one of the central issues in economics. Triangulation of IOTs is one such method that facilitates summarization and analysis of data. This paper proposes a new method to triangulate IOTs based on mixed integer programs (MIPs) for examining production structures of economies, and conducting inter-temporal and inter-regional comparisons.

Primary, secondary, and tertiary sectors in an IOT in its original form are traditionally arranged in the aforementioned order. An IOT of sufficiently detailed sector classification has many zero elements and it is a sparse matrix because most sectors require product inputs from a limited number of sectors. Therefore, an IOT may be triangulated, implying that most elements in the upper triangular part are zeros, by rearranging the sectors based on degrees of fabrication. Triangulation arrangement is fairly straightforward for sectors among which relative degrees of fabrication are clearly defined based on expert knowledge of industrial technologies. For instance, the automotive sector is followed by motor vehicle parts sectors in a triangulated table because an automobile is more highly fabricated than its parts. However, the relative degrees of fabrication among some sectors may not always be clearly defined. In all such cases, an IOT can still be triangulated approximately, for example, by solving an optimization problem. The ordering of sectors in an IOT triangulated in this manner can be interpreted as a descending order of degrees of fabrication, by analogy with the relationship between the automotive sector and motor vehicle parts sectors. This paper proposes a new method of triangulation based on optimization problems.

Leontief (1963, p. 151) traces back the history of triangulation of IOTs to the research by Marshall K. Wood, George D. Danzig, and their associates in Project Scoop of the U.S. Air Force during the late 1940s. Their original motivation in rearranging sectors was to reduce computational burden for solving a system of linear equations. However, they also found that a triangulated IOT reveals structural characteristics of the economy that it represents. Moreover, the major present day utility of triangulation is to serve as a tool for analyzing the production structure of an economy, since solving an optimization problem for triangulation is a much more computer-intensive task than solving a system of linear equations. As Korte and Oberhofer (1970, Sect. 6) summarized, the major applications of triangulated IOTs that contribute to practical economic decision making include studying the production structure of economies, serving as a device to compare various economies, examining sectors that influence 
business cycles and economic growth, reducing computational burden of solving systems of linear equations, and enhancing forecasting and economic planning.

In addition to the applications summarized by Korte and Oberhofer (1970), triangulated IOTs lend themselves to useful analysis on at least two counts in the contemporary context. First, graphical visualization of a triangulated IOT is useful in identifying inter-sectoral dependence. Triangulation greatly improves and enhances the readability of a graphically represented IOT and aids in obtaining the complete picture of inter-sectoral transactions with little loss of information for each transaction. Nakamura et al. (2011) provide an example of visualizing triangulated IOTs for analyzing inter-sectoral flows of iron and steel in passenger car production. The ordering of sectors in triangulated IOTs can also be used to determine the positioning of sectors in a flow diagram. Nakamura et al. (2011) and Nakajima et al. (2013) have shown such drawings through the Sankey diagrams.

Second, the fundamental structure of production discussed by Simpson and Tsukui (1965), its inter-temporal stability, and inter-regional similarity need to be re-investigated. Following the pioneering studies by Chenery and Watanabe (1958), Leontief (1963), and Simpson and Tsukui (1965), a number of studies examined developed and less developed economies through triangulated IOTs and provided empirical evidence that the production structures of these economies are quite similar to each other and fairly stable over time. Korte and Oberhofer (1970), Lamel et al. (1972), Santhanam and Patil (1972), Song (1977), Fukui (1986), Pryor (1994), and Östblom $(1993,1997)$ are some such studies. With recent technological progress engendering factors such as promotion of industrial symbiosis, substitution of materials across sectors, and green product-service system, it is worth checking if these factors have caused a change in hierarchy among sectors. With this background, this paper proposes an extension of the triangulation problem for comparing the production structures of multiple economies.

The remainder of the paper is organized as follows. Section 2 formulates a triangulation problem according to available literature and extends it for developing a new method to compare two or more IOTs. The new method is applied for intertemporal and inter-regional comparisons in Sect. 3. Concluding remarks are outlined in Sect. 4. Computer codes used for implementing the new method are provided in the supplementary material.

\section{Methods}

\subsection{Literature Review}

Most studies such as Simpson and Tsukui (1965) and Fukui (1986) define triangulation of an IOT as an optimization problem to maximize the sum of elements in the lower triangular part by permuting sectors. This is a combinatorial optimization problem known as an NP-hard problem and is difficult to solve (Karp 1972; Charon and Hudry 2007). It offers $n$ ! feasible solutions if there are $n$ sectors. In case the value of $n$ is very small, enumerating all the $n$ ! permutations of sectors and choosing an optimal solution from among them can easily solve the problem. For example, 
if $n=5$, enumerating the $n !=120$ permutations and choosing an optimal solution may resolve the problem. However, such a brute force algorithm works only when the value of $n$ is very small and its application is problematic even in the case of moderate number of sectors such as $n=50$ for which $n ! \approx 3.0 \times 10^{64}$.

Several algorithms specifically designed for the triangulation problem have been developed and proposed in the literature. Simpson and Tsukui (1965), Korte and Oberhofer (1970), and Fukui (1986) proposed heuristic algorithms in which they have iterated substitutions of sectors, called ringshift permutations. However, optimal solutions are not necessarily obtained by executing these algorithms. Algorithms with which optimal solutions can be found for problems of moderate size have also been developed. Haltia (1992) and Östblom (1997) proposed an algorithm without ringshift permutations. The triangulation problem is equivalent to the linear ordering problem and more efficient algorithms for generating optimal solutions have been proposed in the literature on operations research (Grötschel et al. 1984a; Laguna et al. 1999; Mitchell and Borchers 2000; Chiarini et al. 2004; Pintea et al. 2009). Mitchell and Borchers (2000) have noted that an exact solution to a linear ordering problem with 250 objects or sectors can be obtained.

Because the triangulation problem can be represented as an integer program (IP) as explained in Grötschel et al. (1984a, 1984b) and Chiarini et al. (2004), it can be solved, at least approximately, by a general-purpose algorithm for IPs implemented in currently available software. It can be said that most general-purpose algorithms are less efficient than special algorithms. However, general-purpose algorithms, unlike special algorithms, can be applied even when the original problem is extended or modified, for example, by adding constraints or changing the objective function. This paper utilizes an IP representation of the triangulation problem for comparing the production structures of multiple economies.

Inter-temporal or inter-regional comparisons of two (or more) IOTs have been used to address interesting and important issues in economics since the early studies such as those undertaken by Chenery and Watanabe (1958). In addition to comparing the entire sequences of sectors in two triangulated IOTs, the Spearman rank correlation coefficient has been used to quantify the similarity between the two sequences (Chenery and Watanabe 1958; Grötschel et al. 1984b; Fukui 1986; Östblom 1993). As Grötschel et al. (1984b) and Östblom (1997) have pointed out, although the optimal sequences of sectors may appear to be quite different, their underlying structures might be similar. This is because typically, an optimal solution to the triangulation problem is not unique. The non-uniqueness of optimal solutions inherently indicates a basic characteristic of the production structure in terms of sectors. For example, (a) the automotive sector is followed by (e) engine- and (t) tire-producing sectors in a triangulated table. Optimal solutions to the triangulation problem are either $(\mathrm{a}) \rightarrow(\mathrm{e}) \rightarrow(\mathrm{t})$ or $(\mathrm{a}) \rightarrow(\mathrm{t}) \rightarrow(\mathrm{e})$. If two IOTs are individually triangulated and the rank correlation coefficient is calculated without carefully considering the optimal rankings or triangulated tables, the result is more likely to be misinterpreted. It is useless to discuss the difference between the two ordering, (a) $\rightarrow$ $(\mathrm{e}) \rightarrow(\mathrm{t})$ and $(\mathrm{a}) \rightarrow(\mathrm{t}) \rightarrow(\mathrm{e})$, in the example. This paper proposes a new method for comparing the structures of multiple economies, which does not suffer from the non-uniqueness of optimal solutions and is consistent with the maximization of the Kendall rank correlation coefficient. The method is presented in detail in Sect. 2.3. 


\subsection{Definition and Representations of the Triangulation Problem}

Let there be $n$ industrial sectors and suppose that our target is to triangulate an $n \times n$ matrix $\mathbf{A}=\left(A_{i j}\right)$ that describes inter-dependence among sectors. We do not specify this matrix in more detail here, and simply call it an IOT in this section. The next section outlines this aspect in greater detail. We define the set of natural numbers referring to $n$ sectors as $N=\{1, \ldots, n\}$. We then denote a permutation of $n$ sectors by $\pi=(\pi(1), \ldots, \pi(n))$ and the set of all permutations of sectors by $\Pi$. Given an arbitrary permutation $\boldsymbol{\pi} \in \Pi$, let $\mathbf{A}(\boldsymbol{\pi})=\left(A_{i j}(\boldsymbol{\pi})\right)$ denote the IOT in which the sectors are permuted according to $\boldsymbol{\pi}$. This is written as follows:

$$
A_{i j}(\boldsymbol{\pi})=A_{\pi(i) \pi(j)} \quad(i, j \in N) .
$$

The triangulation problem is formulated as a combinatorial optimization problem:

$$
\begin{array}{ll}
\text { maximize } & \ell(\mathbf{A}(\boldsymbol{\pi})) \\
\text { subject to } & \boldsymbol{\pi} \in \Pi,
\end{array}
$$

where $\ell(\mathbf{M})=\sum_{i>j} M_{i j}$ for any $n \times n$ matrix $\mathbf{M}=\left(M_{i j}\right)$, representing the sum of elements in the lower triangular part.

An index called the degree of linearity has been used in the literature (e.g., Fukui 1986) to represent how well an IOT is triangulated. Given an IOT, the degree of linearity of a permutation $\pi$ is defined as follows:

$$
\lambda(\mathbf{A}(\boldsymbol{\pi}))=\frac{\sum_{i>j} A_{i j}(\boldsymbol{\pi})}{\sum_{i \neq j} A_{i j}(\boldsymbol{\pi})}=\frac{\ell(\mathbf{A}(\boldsymbol{\pi}))}{\sum_{i \neq j} A_{i j}} .
$$

The numerator is the same as the objective function of the triangulation problem described by (2). The denominator is the sum of all the off-diagonal elements. Note that a permutation $\pi$ that maximizes the objective function of the triangulation problem $\ell(\mathbf{A}(\boldsymbol{\pi}))$, also maximizes the degree of linearity $\lambda(\mathbf{A}(\boldsymbol{\pi}))$, because the denominator does not depend on $\pi$.

Let us introduce the following $n \times n$ matrix of binary variables, $\mathbf{X}=\left(X_{i j}\right)$ :

$$
X_{i j}=1\left\{\pi^{-1}(i) \geq \pi^{-1}(j)\right\} \quad(i, j \in N),
$$

where $1\{\cdot\}$ is the indicator function such that $1\{P\}=1$ if the proposition $P$ is true, and $1\{P\}=0$ otherwise. Given a permutation $\pi, \pi(p)$ represents the sector at the $p$ th position and $\pi^{-1}(s)$ represents the position at which sector $s$ is placed, where "sector $s$ " refers to the $s$ th sector in the original ordering. Note that $X_{i i}=1$ for every $i \in N, X_{i j}=1$ if sector $j$ precedes sector $i$ in the permutation $\pi$, and $X_{i j}=0$ otherwise. In other words, $A_{i j}$ is located in the lower triangular part or on the main diagonal of $\mathbf{A}(\boldsymbol{\pi})$ if $X_{i j}=1$. The following equality thus holds:

$$
\ell(\mathbf{A}(\boldsymbol{\pi}))=\sum_{i=1}^{n} \sum_{j=1}^{n} A_{i j} X_{i j}-\sum_{i=1}^{n} A_{i i} .
$$


It is known that the triangulation problem described in (2) can be represented as the following IP (deCani 1969; Grötschel et al. 1984a; Chiarini et al. 2004):

$$
\begin{array}{ll}
\operatorname{maximize} & \sum_{i=1}^{n} \sum_{j=1}^{n} A_{i j} X_{i j}-\sum_{i=1}^{n} A_{i i} \\
\text { subject to } & X_{i i}=1 \quad(i \in N), \\
& X_{i j}+X_{j i}=1 \quad(i<j ; i, j \in N), \\
& 0 \leq X_{i j}+X_{j k}-X_{i k} \leq 1 \quad(i<j<k ; i, j, k \in N), \\
& X_{i j} \in\{0,1\} \quad(i, j \in N) .
\end{array}
$$

Alternatively, it can also be represented as the following IP:

$$
\begin{array}{ll}
\operatorname{maximize} & \sum_{i=1}^{n-1} \sum_{j=i+1}^{n}\left\{\left(A_{i j}-A_{j i}\right) X_{i j}+A_{j i}\right\} \\
\text { subject to } & 0 \leq X_{i j}+X_{j k}-X_{i k} \leq 1 \quad(i<j<k ; i, j, k \in N), \\
& X_{i j} \in\{0,1\} \quad(i<j ; i, j \in N) .
\end{array}
$$

More specifically, the last representation is a $\{0,1\}$-program, which has $n(n-1) / 2$ binary variables and $n(n-1)(n-2) / 3$ inequality constraints.

Given that an optimal solution is obtained for (7), the corresponding optimal permutation $\pi$ can then be derived by the following equation:

$$
\pi^{-1}(i)=\sum_{j=1}^{n} X_{i j} \quad(i \in N),
$$

where $X_{i i}=1(i \in N)$ and $X_{j i}=1-X_{i j}(i<j ; i, j \in N)$.

2.3 Extension of the Triangulation Problem for Comparing Input-Output Tables

Suppose that we have IOTs for $n_{T}$ time periods, $\mathbf{A}^{(t)}=\left(A_{i j}^{(t)}\right)(t \in T)$, where $T=\left\{1, \ldots, n_{T}\right\}$ and superscript " $(t)$ " indicates the time period (the method proposed in this section can be applied for inter-regional comparisons; however, this section focuses on inter-temporal comparisons). Suppose that we have solved IP (7) with $\mathbf{A}=\mathbf{A}^{(t)}$ for all time periods and obtained an optimal solution $\bar{X}_{i j}^{(t)}$, the corresponding optimal permutation $\overline{\boldsymbol{\pi}}_{(t)}$, and the optimal value $\bar{M}_{(t)}$ (maximized objective value) of the program. We propose to find $n_{T}$ sequences of sectors that are mutually as close as possible, guaranteeing that best degrees of linearity are attained. The problem for $n_{T}=2$ and $T=\{1,2\}$ can be written as follows, with the concept of "difference" allowed to be ambiguous:

$$
\begin{array}{ll}
\text { minimize } & \text { difference between } \boldsymbol{\pi}_{(1)} \text { and } \boldsymbol{\pi}_{(2)} \\
\text { subject to } & \ell\left(\mathbf{A}^{(t)}\left(\boldsymbol{\pi}_{(t)}\right)\right)=\bar{M}_{(t)} \quad(t \in T), \\
& \boldsymbol{\pi}_{(t)} \in \Pi \quad(t \in T) .
\end{array}
$$


Let us define the difference between $\boldsymbol{\pi}_{(1)}$ and $\boldsymbol{\pi}_{(2)}$ as the sum of absolute differences between the elements of $\mathbf{X}^{(1)}$ and $\mathbf{X}^{(2)}$, that is, $\sum_{i=1}^{n-1} \sum_{j=i+1}^{n}\left|X_{i j}^{(1)}-X_{i j}^{(2)}\right|$. The advantage of this difference over others such as the sum of squared differences will be discussed later. We now introduce new variables, $U_{i j}, V_{i j}(i<j ; i, j \in N)$, such that

$$
U_{i j}-V_{i j}=X_{i j}^{(1)}-X_{i j}^{(2)}, \quad U_{i j} \geq 0, V_{i j} \geq 0(i<j ; i, j \in N) .
$$

Note that $\left|X_{i j}^{(1)}-X_{i j}^{(2)}\right|=U_{i j}+V_{i j}$ if $U_{i j} V_{i j}=0$ and (10) hold for any pair of $X_{i j}^{(1)}$ and $X_{i j}^{(2)}$. This is a well-known technique for dealing with absolute values in the field of operations research.

By employing this technique, we make the program (9) concrete and propose the following MIP for comparing hierarchies among sectors in two IOTs:

$$
\begin{array}{ll}
\operatorname{minimize} & \sum_{i=1}^{n-1} \sum_{j=i+1}^{n}\left(U_{i j}+V_{i j}\right) \\
\text { subject to } \quad & 0 \leq X_{i j}^{(t)}+X_{j k}^{(t)}-X_{i k}^{(t)} \leq 1 \quad(i<j<k ; i, j, k \in N ; t \in T), \\
& U_{i j}-V_{i j}=X_{i j}^{(1)}-X_{i j}^{(2)} \quad(i<j ; i, j \in N), \\
& \sum_{i=1}^{n-1} \sum_{j=i+1}^{n}\left\{\left(A_{i j}^{(t)}-A_{j i}^{(t)}\right) X_{i j}^{(t)}+A_{j i}^{(t)}\right\}=\bar{M}_{(t)} \quad(t \in T), \\
& X_{i j}^{(t)} \in\{0,1\} \quad(i<j ; i, j \in N ; t \in T), \\
& U_{i j} \geq 0, \quad V_{i j} \geq 0 \quad(i<j ; i, j \in N) .
\end{array}
$$

The first and fourth constraints form the same set of constraints in (7) and correspond to the constraints $\boldsymbol{\pi}_{(t)} \in \Pi(t \in T)$ in (9). $\mathbf{X}^{(1)}=\left(X_{i j}^{(1)}\right)$ and $\mathbf{X}^{(2)}=\left(X_{i j}^{(2)}\right)$ consistently represent two permutations of sectors. The second and fifth constraints and the objective function compose the technique to minimize the difference between $\boldsymbol{\pi}_{(1)}$ and $\pi_{(2)}$, or $\sum_{i=1}^{n-1} \sum_{j=i+1}^{n}\left|X_{i j}^{(1)}-X_{i j}^{(2)}\right|$. The third constraint corresponds to the constraints $\ell\left(\mathbf{A}^{(t)}\left(\boldsymbol{\pi}_{(t)}\right)\right)=\bar{M}_{(t)}(t \in T)$ in (9) and guarantees that best degrees of linearity are attained. It should be noted that the nonlinear constraint $U_{i j} V_{i j}=0$ is not necessary in the program (11) in the sense that optimal solutions always satisfy the constraint because of the characteristics of its objective function. ${ }^{1}$ Therefore, the linearity of the program, except for the binary constraints on $X_{i j}^{(t)}$, is maintained.

The advantage of program (11), a specific form of the general program (9), over other forms is at least twofold. First, the objective function and constraints, except for integralities, are linear in variables. This linearity is almost a prerequisite because we

\footnotetext{
${ }^{1}$ Suppose that $U_{i j} V_{i j} \neq 0$ for some feasible solution for (11). By letting $W_{i j}=\min \left\{U_{i j}, V_{i j}\right\}$ and replacing $\left(U_{i j}, V_{i j}\right)$ with $\left(U_{i j}-W_{i j}, V_{i j}-W_{i j}\right)$, we can construct another feasible solution which has a smaller objective value and satisfies the nonlinear constraint as $\left(U_{i j}-W_{i j}\right)\left(V_{i j}-W_{i j}\right)=0$.
} 
use a general algorithm, rather than develop specialized algorithms. Therefore, we do not specify the difference between $\boldsymbol{\pi}_{(1)}$ and $\boldsymbol{\pi}_{(2)}$ as a nonlinear formula such as the sum of squared differences between rankings.

Second, MIP (11) is consistent with the Kendall rank correlation coefficient. Note that for any pair $(i, j)$ such that $i \neq j(i, j \in N)$,

$$
X_{i j}^{(1)}-X_{i j}^{(2)}= \begin{cases}1 & \text { if } \pi_{(1)}^{-1}(i)>\pi_{(1)}^{-1}(j) \text { and } \pi_{(2)}^{-1}(i)<\pi_{(2)}^{-1}(j), \\ -1 & \text { if } \pi_{(1)}^{-1}(i)<\pi_{(1)}^{-1}(j) \text { and } \pi_{(2)}^{-1}(i)>\pi_{(2)}^{-1}(j), \\ 0 & \text { otherwise, }\end{cases}
$$

because $X_{i j}^{(t)}=1\left\{\pi_{(t)}^{-1}(i)>\pi_{(t)}^{-1}(j)\right\}(i \neq j ; i, j \in N, t \in T)$ according to (4). Thus, the objective function of the proposed program (11), $Q=\sum_{i<j}\left|X_{i j}^{(1)}-X_{i j}^{(2)}\right|=$ $\sum_{i<j}\left(U_{i j}+V_{i j}\right)$, can be interpreted as the number of pairs $(i, j)$ of sectors that disagree in the two rankings. Note also that the Kendall rank correlation coefficient between $\boldsymbol{\pi}_{(1)}$ and $\boldsymbol{\pi}_{(2)}$ is given by $\tau\left(\boldsymbol{\pi}_{(1)}, \boldsymbol{\pi}_{(2)}\right)=1-4 Q / n(n-1)$ (see, for example, Kendall and Gibbons 1990, p. 5). Thus, it has been shown that the proposed MIP (11) maximizes the Kendall rank correlation coefficient between two sequences of sectors, guaranteeing that best degrees of linearity are attained. A variant of MIP (11) that is consistent with the Spearman rank correlation coefficient can also be formulated by specifying its objective function as the sum of squared differences between the rankings, $\sum_{i=1}^{n}\left(\pi_{(1)}^{-1}(i)-\pi_{(2)}^{-1}(i)\right)^{2}$. However, we will not employ this nonlinear formulation because a linear formulation is preferred to a nonlinear formulation for our study.

MIP (11) for $n_{T}=2$ can be generalized for cases of $n_{T} \geq 3$ as follows:

$$
\begin{array}{ll}
\operatorname{minimize} & \sum_{s=1}^{n_{T}-1} \sum_{t=s+1}^{n_{T}} \sum_{i=1}^{n-1} \sum_{j=i+1}^{n}\left(U_{i j}^{(s t)}+V_{i j}^{(s t)}\right) \\
\text { subject to } \quad & 0 \leq X_{i j}^{(t)}+X_{j k}^{(t)}-X_{i k}^{(t)} \leq 1 \quad(i<j<k ; i, j, k \in N ; t \in T), \\
& U_{i j}^{(s t)}-V_{i j}^{(s t)}=X_{i j}^{(s)}-X_{i j}^{(t)} \quad(i<j ; i, j \in N ; s<t ; s, t \in T), \\
& \sum_{i=1}^{n-1} \sum_{j=i+1}^{n}\left\{\left(A_{i j}^{(t)}-A_{j i}^{(t)}\right) X_{i j}^{(t)}+A_{j i}^{(t)}\right\}=\bar{M}_{(t)} \quad(t \in T), \\
& X_{i j}^{(t)} \in\{0,1\} \quad(i<j ; i, j \in N ; t \in T), \\
& U_{i j}^{(s t)} \geq 0, \quad V_{i j}^{(s t)} \geq 0 \quad(i<j ; i, j \in N ; s<t ; s, t \in T) .
\end{array}
$$

In this generalization, superscript "(st)" is introduced for $U_{i j}$ and $V_{i j}$ to indicate different time periods for comparison. For formulating the objective function in (13), sums of absolute differences between elements of $\mathbf{X}^{(s)}$ and $\mathbf{X}^{(t)}$ for all $n_{T}\left(n_{T}-\right.$ 1)/2 pair-wise comparisons are added up. Recall that minimization of the sum of absolute differences $Q^{(s t)}=\sum_{i<j}\left|X_{i j}^{(s)}-X_{i j}^{(t)}\right|$ is consistent with maximization of the Kendall rank correlation coefficient $\tau\left(\boldsymbol{\pi}_{(s)}, \boldsymbol{\pi}_{(t)}\right)=1-4 Q^{(s t)} / n(n-1)$ between 
$\boldsymbol{\pi}_{(s)}$ and $\boldsymbol{\pi}_{(t)}$. Therefore, minimization in (13) is consistent with maximization of the simple arithmetic mean of the Kendall rank correlation coefficients for all pairwise comparisons, that is, $c=\left(n_{T}\left(n_{T}-1\right) / 2\right)^{-1} \sum_{s<t} \tau\left(\boldsymbol{\pi}_{(s)}, \boldsymbol{\pi}_{(t)}\right)$. This is one of the multivariate generalizations of the pair-wise Kendall rank correlation coefficient studied by Joe (1990, p. 21).

The following MIP should also be useful when a slight deterioration of the degree of linearity is acceptable in comparing the optimal ordering of sectors:

$$
\begin{array}{ll}
\text { minimize } & \sum_{s=1}^{n_{T}-1} \sum_{t=s+1}^{n_{T}} \sum_{i=1}^{n-1} \sum_{j=i+1}^{n}\left(U_{i j}^{(s t)}+V_{i j}^{(s t)}\right) \\
\text { subject to } \quad & 0 \leq X_{i j}^{(t)}+X_{j k}^{(t)}-X_{i k}^{(t)} \leq 1 \quad(i<j<k ; i, j, k \in N ; t \in T), \\
& U_{i j}^{(s t)}-V_{i j}^{(s t)}=X_{i j}^{(s)}-X_{i j}^{(t)} \quad(i<j ; i, j \in N ; s<t ; s, t \in T), \\
& \sum_{i=1}^{n-1} \sum_{j=i+1}^{n}\left\{\left(A_{i j}^{(t)}-A_{j i}^{(t)}\right) X_{i j}^{(t)}+A_{j i}^{(t)}\right\} \geq \alpha \bar{M}_{(t)} \quad(t \in T), \\
& X_{i j}^{(t)} \in\{0,1\} \quad(i<j ; i, j \in N ; t \in T), \\
& U_{i j}^{(s t)} \geq 0, \quad V_{i j}^{(s t)} \geq 0 \quad(i<j ; i, j \in N ; s<t ; s, t \in T),
\end{array}
$$

where $\alpha$ is a constant such that $0<\alpha \leq 1$. MIPs (13) and (14) are the same, except for the difference in the third constraint. The smaller the value of $\alpha$ is, the more concordant optimal ordering of sectors is obtained at the cost of degrees of linearity. Note that MIP (13), by construction, is equivalent to MIP (14) when $\alpha=1$.

\section{Empirical Applications}

There are several possible choices for the target matrix to triangulate. For example, the flow matrix $\mathbf{Z}=\left(Z_{i j}\right)$, where $Z_{i j}$ refers to the intermediate flow from sector $i$ to sector $j$; the input coefficient matrix $\mathbf{A}=\left(A_{i j}\right)=\left(Z_{i j} / x_{j}\right)$, where $x_{j}$ refers to the output of sector $j$; the Leontief inverse matrix $\mathbf{L}=(\mathbf{I}-\mathbf{A})^{-1}$; the output coefficient matrix $\mathbf{B}=\left(B_{i j}\right)=\left(Z_{i j} / x_{i}\right)$; and the Ghosh inverse matrix $\mathbf{G}=(\mathbf{I}-\mathbf{B})^{-1}$. Although comparison among these matrices is beyond the scope of this paper, it is worth giving a comment on the relationship between direct and indirect linkages. Indirect linkages represented by $\mathbf{L}$ are properly considered even if the target matrix to triangulate is $\mathbf{A}$, in which only direct linkages are represented by positive elements, because all sectors are simultaneously arranged in a sequence. See Korte and Oberhofer (1970, Sect. 6) for a discussion of how the optimal ordering of sectors and its interpretations vary with different matrices to be triangulated. We chose the input coefficient matrix $\mathbf{A}$ for our empirical applications because the purpose is to compare production systems from a technological perspective and/or fundamental structures of economy, factors that are independent of the final demand (Leontief 1953; Simpson and Tsukui 1965).

Xpress Mosel language version 3.2.3 and Xpress Optimizer version 22.01.09 were used to solve IPs and MIPs. Calculations were performed on a workstation with two 
Table 1 Inter-temporal comparison of the Japanese economy between 1995 and 2005

\begin{tabular}{|c|c|c|c|c|c|c|}
\hline & \multicolumn{2}{|c|}{ Degrees of linearity } & \multicolumn{2}{|c|}{ Individual } & \multicolumn{2}{|c|}{ Simultaneous } \\
\hline & 1995 & 2005 & $\rho$ & $\tau$ & $\rho$ & $\tau$ \\
\hline Without trimming & 0.9100 & 0.9090 & 0.9579 & 0.9126 & 0.9666 & 0.9437 \\
\hline With trimming & 0.9481 & 0.9479 & 0.8751 & 0.7329 & 0.9470 & 0.9018 \\
\hline
\end{tabular}

Note: Rank correlation coefficients are in the columns labeled "Individual" and "Simultaneous." "Individual" refers to the results of IP (7) and "Simultaneous" refers to results of MIP (11). $\rho$ and $\tau$ refer to the Spearman and Kendall rank correlation coefficients, respectively

Source: Own calculation

Intel ${ }^{\circledR}$ Xeon ${ }^{\circledR}$ CPUs of $2.27 \mathrm{GHz}$ and 24 GB RAM. The operating system and software were of 64 bits.

\subsection{The Japanese Economy in 1995 and 2005: Inter-temporal Comparison}

Data for the input coefficient matrices representing the production structure of the Japanese economy in 1995 and 2005 were obtained from the fixed-price tables of the Japanese Linked Input-Output Tables (SB-MIAC 2011). This particular study period is chosen because it is covered by the latest available linked IOTs. In addition to these input coefficient matrices, we also utilized "trimmed" versions of the input coefficient matrices to check the robustness of our analysis. The trimmed input coefficient matrix was constructed by setting $A_{i j}=0$ if $A_{i j}<1 / n(i, j \in N)$, according to Simpson and Tsukui (1965). Typically, the larger elements are likely to be estimated more precisely, while the smaller elements possibly include more noise. The trimmed matrices are expected to emphasize significant inter-dependencies between sectors or highlight the features of production structures. The number of sectors is $n=102$. The number of non-zero elements of the trimmed matrix is 1104 for 1995 and 1135 for 2005, while that for the input coefficient matrix without trimming is 6321 for 1995 and 6326 for 2005 . For each year, the sum of all elements of the trimmed matrix is about $84 \%$ of the sum of all elements of the matrix without trimming, while the number of non-zero elements of the trimmed matrix is considerably smaller than that of the matrix without trimming. Therefore, it can be observed that trimming effectively highlights the essential features of production structures.

Both the matrices were effectively triangulated, without trimming, by solving IP (7): the degrees of linearity were about 0.91 for each year, as shown in Table 1. The trimmed matrices were also triangulated almost perfectly and the degrees of linearity were about 0.95 , as shown in Table 1 . These values close to unity imply that the inter-dependence among sectors can be summarized as a nearly uni-directional hierarchy. Thus, there is little multi-directional dependence such as feedback loops with substantial inter-sectoral transactions.

To summarize the similarity of optimal rankings of sectors in 1995 and 2005, we calculated the Spearman rank correlation coefficient $\rho$ and the Kendall rank correlation coefficient $\tau$. As shown in the column labeled "Individual" in Table 1, the rank correlation coefficients are very large for the matrices without trimming: $\rho=0.958$ 
and $\tau=0.913$. This result indicates that the production structure revealed by triangulation is fairly stable during the period $1995-2005$. On the contrary, weaker correlation coefficients were obtained with the trimmed matrices: $\rho=0.875$ and $\tau=0.733$. This result implies that either the production structure has gone through a mild change during 1995-2005 or a stable structure cannot be revealed due to non-uniqueness of optimal solutions to the triangulation problem. It is found that it is the latter in this case. Much stronger correlation such as $\rho=0.947$ and $\tau=0.902$ was obtained by solving MIP (11), as shown in Table 1 . Therefore, by utilizing the new method proposed in Sect. 2, we could identify a fairly stable production structure in Japan during 1995-2005.

The optimal ordering of sectors obtained by solving IP (7) and MIP (11) for the trimmed matrices are shown in Figs. 1 and 2, respectively, in the form of a migration diagram (Grötschel et al. 1984b). Figure 1 has much more crossings than Fig. 2. Note that the ordering of sectors in Fig. 2 was obtained by minimizing the number of crossings, or equivalently, by maximizing the Kendall rank correlation coefficient between orderings in 1995 and 2005, keeping the degrees of linearity equal to optimal levels. The two diagrams clearly show that a comparison based on IP (7) is strongly influenced by the non-uniqueness of optimal solutions.

As shown in Fig. 2, it is found that the top 24 sectors agree with each other in the two rankings. This implies that the drastic change in hierarchies, which appears in the form of several crossings in the upper half of Fig. 1, is not meaningful. The top 50 sectors approximately remain in top positions over time. Such stability in rankings is not limited to top-ranked sectors only. The difference in ranking for two years is less than 10 for 95 sectors.

Figure 2 shows that sector \#87 "Public administration," sector \#83 “Communication," and sector \#85 "Information services" drastically changed their rankings during 1995-2005. Only sector \#102 "Activities not elsewhere classified" purchases the output of "Public administration," and the input coefficient $A_{87,102}$ drastically increased from 0.076 to 0.279 during 1995-2005. Thus, "Public administration" sector was located just after "Activities not elsewhere classified" sector in the 2005 rankings. It is inherently difficult to infer the economic interpretation of this result because of the highly miscellaneous nature of sector \#102.

Sector \#83 "Communication" and sector \#85 "Information services" are located in the middle of the optimal sequence (i.e., at the 59th and 54th positions), respectively, in the ordering for 1995. On the contrary, these sectors are located at the bottom of the sequence (i.e., at the 99th and 93rd positions), respectively, in 2005. This indicates that the importance of these infrastructural service industries has gone up during 1995-2005. On an average, the input coefficients representing purchase of "Communication" and "Information services" per unit of output of industrial sectors have substantially increased by $34 \%$ and $96 \%$, respectively, over the study period.

Except for these drastic changes in sector ranking, the fundamental structure of the Japanese economy was fairly stable during 1995-2005, as shown in Fig. 2. Personal service sectors (e.g., medical service and health care, accommodations, and restaurants) and final products manufacturing sectors (e.g., machinery, and electric and electrical equipment) are located at the top positions. The products of these sectors are mostly purchased by the final demand sector. Business service sectors (e.g., 


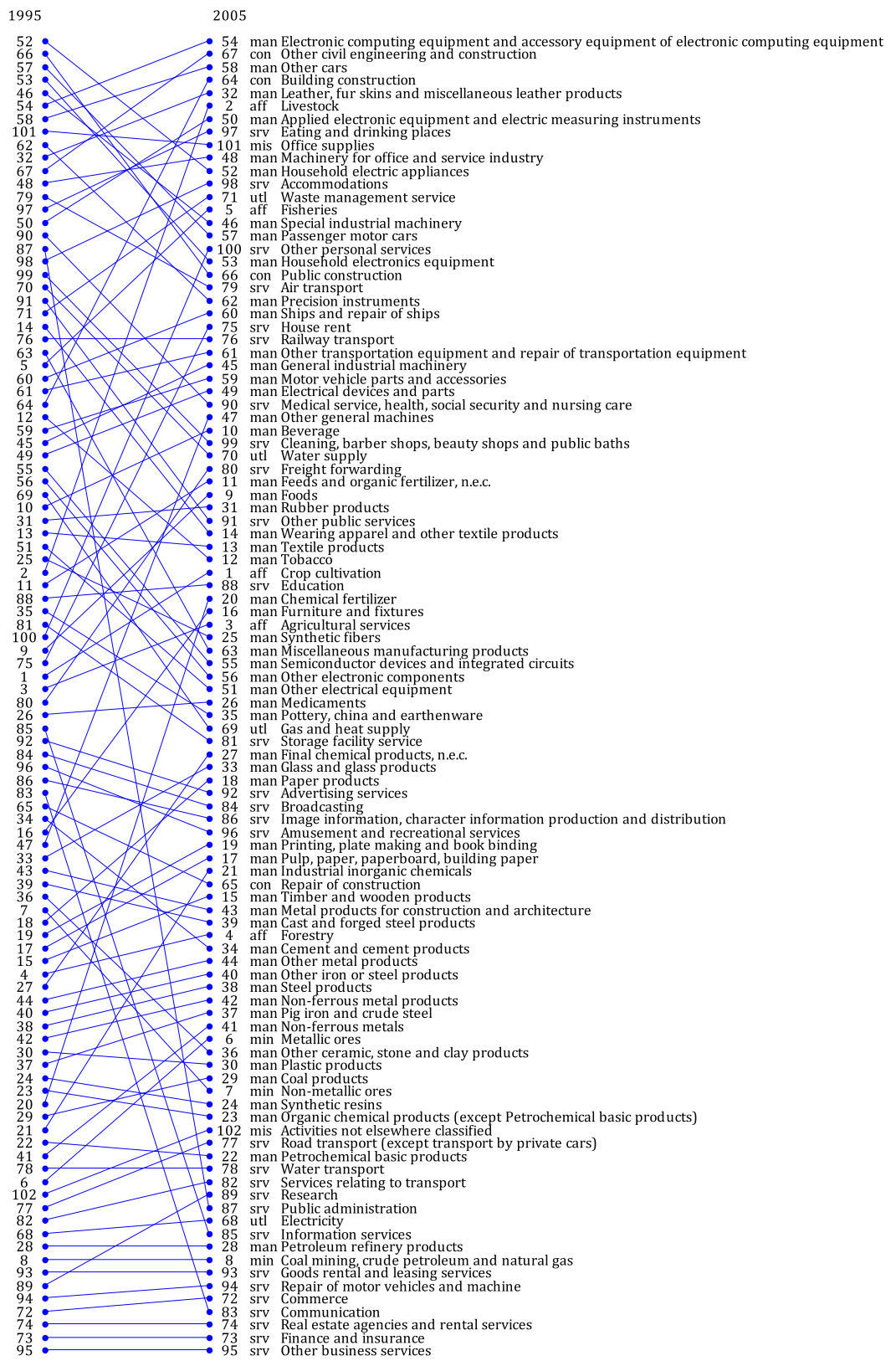

Fig. 1 Inter-temporal comparison of the Japanese economy between 1995 and 2005 based on individual triangulation. Note: The trimmed input coefficient matrices are triangulated individually by IP (7). Threecharacter codes represent the following aggregated sector classification. aff: Agriculture, forestry and fishery; min: Mining; man: Manufacturing; utl: Utilities; con: Construction; ser: Services; mis: Miscellaneous 


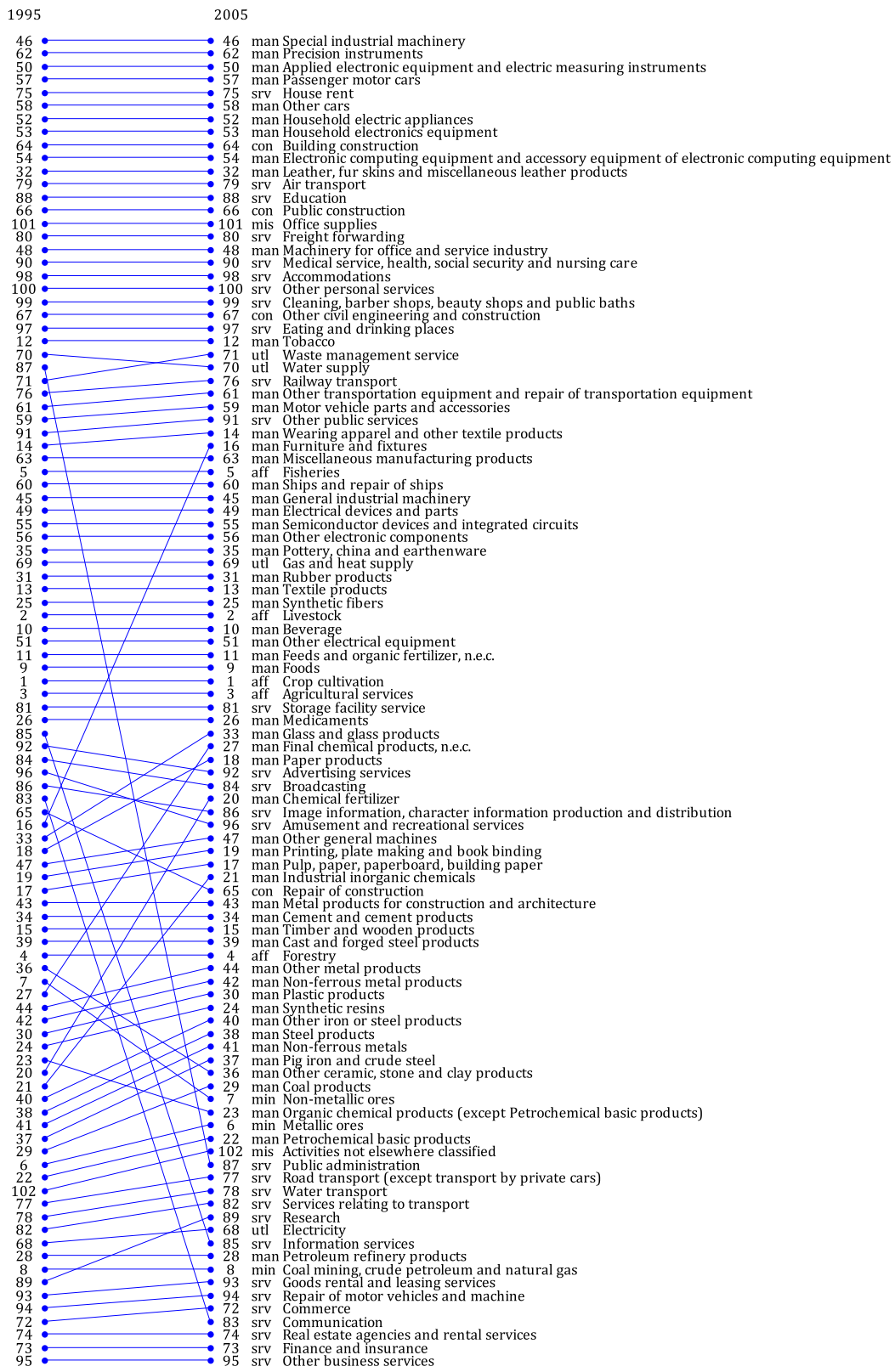

Fig. 2 Inter-temporal comparison of the Japanese economy between 1995 and 2005 based on simultaneous triangulation. Note: The trimmed input coefficient matrices are triangulated simultaneously by MIP (11). See also notes to Fig. 1 
transportation, research, commerce, and finance and insurance), mining sectors, utility sectors (electricity), and basic products manufacturing sectors (e.g., petroleum refinery and petrochemical basic products, coal products, and metal products) are located at the bottom positions. The products of these sectors except for mining sectors are purchased by most industry sectors, while the products of mining sectors are mostly purchased by the basic products manufacturing sectors. The sectors located in the middle positions are parts manufacturing sectors (e.g., motor vehicle parts, electrical devices and parts, and semiconductor devices), light industry sectors (e.g., textile products, wooden products, and paper products), and agriculture, forestry, and fishery sectors.

This fundamental structure of the Japanese economy observed by triangulation is very similar to the fundamental structure found by Simpson and Tsukui (1965), except for the drastic changes in sector ranking mentioned above. From a point of view of empirical analysis, it is found that the fundamental structure of the Japanese economy has been fairly stable over the last decades, and it is slightly changing because of the expansion of information and communication technology. From a point of view of method development, the new method for triangulation based on MIP (11) is very useful to clearly highlight exceptional difference in sector ranking between time periods.

\subsection{Chinese, Japanese, and the U.S. Economies in 2009: Inter-regional Comparison}

Domestic direct requirement matrices for the 2009 national input-output tables of China, Japan, and the U.S. were used for inter-regional comparison. Data were obtained from the World Input-Output Database (WIOD) (Timmer 2012; Dietzenbacher et al. 2013). The year 2009 is the latest available period in WIOD. The number of sectors is $n=35$. The number of non-zero elements is 1085 for China, 1156 for Japan, and 1148 for the U.S. These matrices are not sparse as only 6\%-11\% of 1225 elements are zero. These matrices are relatively denser than data pertaining to 102 sectors outlined in Sect. 3.1 possibly because of higher aggregation of sector classification.

Although the three matrices are not sparse, they were triangulated by solving IP (7). The degrees of linearity were $0.812,0.815$, and 0.873 for China, Japan, and the U.S., respectively, as shown in Table 2. These results are comparable with those reported in the literature. For example, Fukui (1986) showed that the degrees of linearity range from 0.83 to 0.94 for 22- or 29-sector IOTs of India, Italy, Japan, Korea, Norway, and the U.S. for the period 1947-1965.

A comparison of the optimal ordering of sectors indicates the presence of weak similarities among the production structures of the three countries. As shown in the row labeled "Individual" in Table 2, the Kendall rank correlation coefficient ranges from 0.462 to 0.620 , revealing weak similarities. These conclusions are also supported by results obtained from solving MIP (13). For Japan and the U.S., MIP (13) has provided exactly the same optimal ordering as IP (7). For China, the optimal ordering obtained by MIP (13) is the same as the ordering obtained by IP (7), except for four sectors, in which case two sectors are interchanged with their adjacent sectors. Thus, the concordance in ordering of sectors has strengthened by the simultaneous optimization, that is, MIP (13) with respect to these four sectors of China. 
Table 2 Inter-regional comparison among the Chinese, Japanese, and the U.S. economy in 2009

\begin{tabular}{lllll}
\hline Country & \multicolumn{2}{c}{ China (CHN) } & Japan (JPN) & USA \\
\hline Degrees of linearity & \multicolumn{2}{c}{0.8121} & 0.8154 & 0.8732 \\
\hline Kendall $\tau$ & & & & \\
\hline Pair of countries & $(\mathrm{CHN}, \mathrm{JPN})$ & (CHN, USA) & (JPN, USA) & Mean \\
\hline Individual & 0.5866 & 0.4622 & 0.6202 & 0.5563 \\
Simultaneous & 0.5933 & 0.4689 & 0.6202 & 0.5608 \\
$\alpha=0.99$ & 0.8857 & 0.8588 & 0.8387 & 0.8611 \\
$\alpha=0.95$ & 1.0000 & 1.0000 & 1.0000 & 1.0000 \\
\hline
\end{tabular}

Note: "CHN" and "JPN" refer to China and Japan, respectively. The Kendall rank correlation coefficients are in the lower table. "Individual" refers to the results of IP (7) and "Simultaneous" refers to the results of MIP (13) or MIP (14) with $\alpha=1$. The two rows at the bottom refer to the results of MIP (14) with $\alpha=0.99$ and 0.95

Source: Own calculation

MIP (14) was solved to check the possibility of further strengthening of concordance between ordering. The Kendall rank correlation coefficients obtained are shown in the row labeled "Simultaneous" in Table 2. The result of MIP (14) with $\alpha=1$ is the same as that of MIP (13) described above. The result of MIP (14) with $\alpha=0.99$ shows that the Kendall rank correlation coefficient ranges from 0.839 to 0.886 . MIP (14) with $\alpha=0.95$ provides the Kendall rank correlation coefficient value equal to unity, which indicates that the optimal ordering of sectors is common to all the three countries. Therefore, the production structures of China, Japan, and the U.S. can be regarded as very similar if we accept a $5 \%$ loss of degree of linearity.

The optimal ordering of sectors obtained by solving IP (7) and MIP (14) with $\alpha=0.99$ are shown in Figs. 3 and 4, respectively. Note that in Fig. 4, the ordering of sectors was obtained by minimizing the number of crossings drawn in the figure as well as crossings that would appear if sectors of the U.S. were connected with corresponding sectors of China. A comparison of Figs. 3 and 4 shows that production structures of the three countries appear similar on accepting a $1 \%$ loss of degree of linearity.

It is worth pointing out some sectors that cause exceptional dissimilarity among the production structures of the countries. Sector \#2 "Mining and quarrying" in Japan is located at a very higher position as compared to China and the U.S., since the mining and quarrying sector in Japan operates on a much smaller scale than in China and the U.S. Moreover, the positions of service-producing sectors such as sector \#26 "Other supporting and auxiliary transport activities; activities of travel agencies," sector \#27 "Post and telecommunications," and sector \#34 "Other community, social, and personal services" are relatively high in China (13th, 14th, and 15th position, respectively), while they are lower in Japan (17th, 18th, and 22nd position, respectively), and further lower in the U.S. (26th, 27th, and 28th position, respectively). This may indicate that the importance of these sectors as infrastructural service industries is the highest in the U.S., followed by Japan, and least in China. Except for these two 


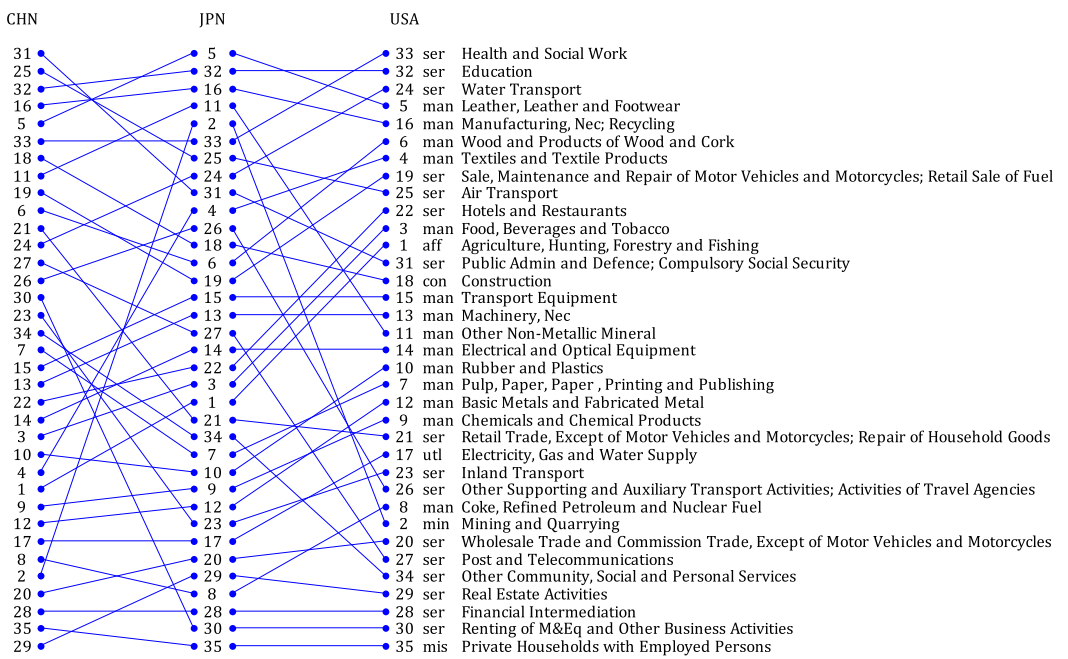

Fig. 3 Sector rankings of China, Japan, and the U.S. based on individual triangulation. Note: The domestic input coefficient matrices are triangulated individually by IP (7). See also notes to Fig. 1

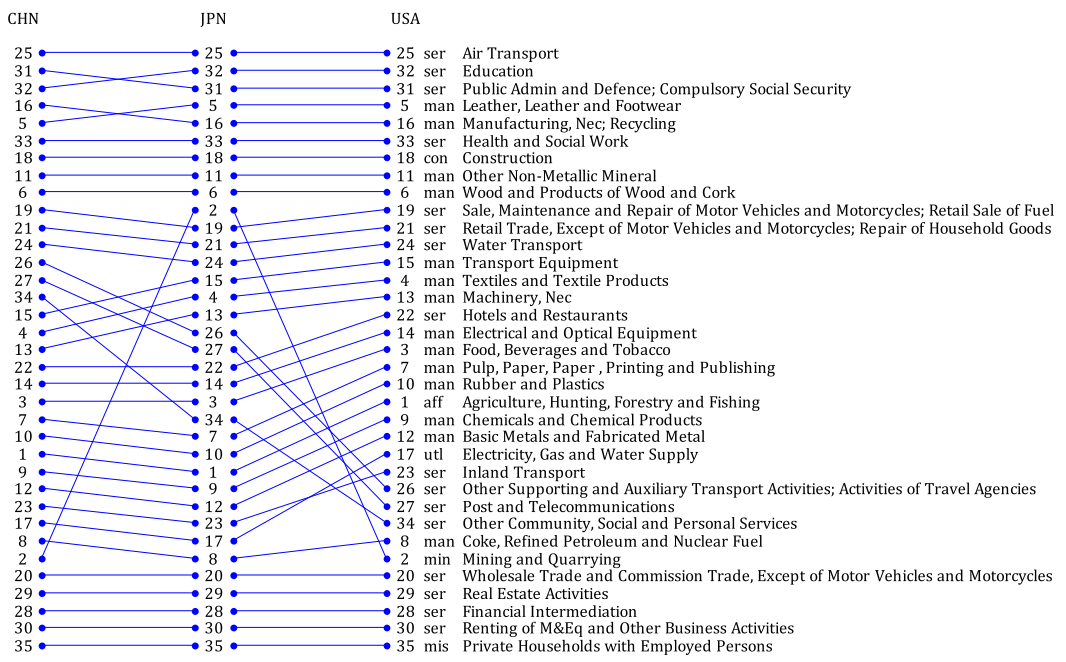

Fig. 4 Sector rankings of China, Japan, and the U.S. based on simultaneous triangulation. Note: The domestic input coefficient matrices are triangulated simultaneously by MIP (14) with $\alpha=0.99$. See also notes to Fig. 1

aspects, the production structures of China, Japan, and the U.S. can be regarded as being very similar, on accepting $1 \%$ loss of degree of linearity.

As shown in Fig. 4, the fundamental structures of the countries found in this section are roughly similar to those of the Japanese economy discussed in Sect. 3.1. Personal service sectors and final products manufacturing sectors are located at the 
top positions, and followed by light industry sectors, basic products manufacturing sectors, utility sectors, mining sectors, and business service sectors.

\section{Concluding Remarks}

This paper has proposed a new method for triangulation of input-output tables (IOTs) by extending an integer-program representation of the triangulation problem for conducting inter-temporal and inter-regional comparisons. The new method provides sequences of sectors that are mutually as close as possible and consistent with maximization of the Kendall rank correlation coefficient. This study demonstrates the utility of the new method by applying it to the Chinese, Japanese, and the U.S. inputoutput tables. Comparisons based on individual application of the existing method for triangulation were found to be strongly influenced by the non-uniqueness of optimal solutions. Consequently, smaller rank correlation was obtained even though the underlying production structures may be very similar. On the contrary, employment of the new method revealed similarities among the production structures of China, Japan, and the U.S. It also enabled investigation of the exceptional dissimilarity between the economies by examining the sectors that were positioned differently.

Future research in this area may apply the new method to larger datasets. Obtaining practically good solutions, instead of optimal solutions, would be useful for a large dataset because it may not be easy to solve large-scale mixed integer programs on personal computers or workstations. Application of the new method to the following datasets is another important area of future research: inter-sectoral material flows (Nakamura et al. 2011; Nakajima et al. 2013), multi-regional input-output tables including carbon, water, and material flows embodied in trade (i.e., carbon, water, and material footprint of transacted goods and services) (Peters et al. 2011; Steen-Olsen et al. 2012; Wiedmann et al. 2013; Tukker and Dietzenbacher 2013), and standard monetary input-output tables. The application will provide a useful tool for graphically visualizing the inter-relationship among sectors from various perspectives.

\section{Competing Interests}

There are no conflicts of interest to declare.

Acknowledgements A preliminary version of this paper was presented at the 19th International InputOutput Conference at Alexandria, Virginia, the U.S. in June 2011. I am grateful to Dr. Richard Wood, anonymous referees, and the editor for their detailed and helpful comments. This research was partially supported by research funds from MEXT KAKENHI (24510059) and the Environment Research and Technology Development Fund (K122024) of the Ministry of the Environment, Japan.

\section{References}

Charon I, Hudry O (2007) A survey on the linear ordering problem for weighted or unweighted tournaments. 4OR 5(1):5-60 
Chenery HB, Watanabe T (1958) International comparisons of the structure of production. Econometrica 26(4):487-521

Chiarini BH, Chaovalitwongse W, Pardalos PM (2004) A new algorithm for the triangulation of inputoutput tables in economics. In: Pardalos PM (ed) Supply chain and finance. World Scientific, Singapore, pp 253-272 (Chap 15)

deCani JS (1969) Maximum likelihood paired comparison ranking by linear programming. Biometrika 56(3):537-545

Dietzenbacher E, Los B, Stehrer R, Timmer M, de Vries G (2013) The construction of world input-output tables in the WIOD project. Econ Syst Res 25(1):71-98

Fukui Y (1986) A more powerful method for triangularizing input-output matrices and the similarity of production structures. Econometrica 54(6):1425-1433

Grötschel M, Jünger M, Reinelt G (1984a) A cutting plane algorithm for the linear ordering problem. Oper Res 32(6):1195-1220

Grötschel M, Jünger M, Reinelt G (1984b) Optimal triangulation of large real world input-output matrices. Stat Hefte 25(1):261-295

Haltia O (1992) A triangularization algorithm without ringshift permutation. Econ Syst Res 3(3):223-234

Joe H (1990) Multivariate concordance. J Multivar Anal 35(1):12-30

Karp RM (1972) Reducibility among combinatorial problems. In: Miller RE, Thatcher JW, Bohlinger JD (eds) Complexity of computer computations. Springer, New York, pp 85-103

Kendall M, Gibbons JD (1990) Rank correlation methods, 5th edn. Edward Arnold, London

Korte B, Oberhofer W (1970) Triangularizing input-output matrices and the structure of production. Eur Econ Rev 1(4):482-511

Laguna M, Marti R, Campos V (1999) Intensification and diversification with elite tabu search solutions for the linear ordering problem. Comput Oper Res 26(12):1217-1230

Lamel J, Richter J, Teufelsbauer W (1972) Patterns of industrial structure and economic development: triangulation of input-output tables of ECE countries. Eur Econ Rev 3(1):47-63

Leontief W (1953) The input-output approach in economic analysis. In: The Netherlands Economic Institute (ed) Input-output relations: proceedings of a conference on inter-industrial relations, Driebergen, Holland, pp 1-23

Leontief W (1963) The structure of development. Sci Am 209(3):148-166

Mitchell JE, Borchers B (2000) Solving linear ordering problems with a combined interior point/simplex cutting plane algorithm. In: Frenk HL, Roos C, Terlaky T, Zhang S (eds) High performance optimization. Kluwer Academic, Dordrecht, pp 349-366 (Chap 14)

Nakajima K, Ohno H, Kondo Y, Matsubae K, Takeda O, Miki T, Nakamura S, Nagasaka T (2013) Simultaneous material flow analysis of nickel, chromium, and molybdenum used in alloy steel by means of input-output analysis. Environ Sci Technol 47(9):4653-4660

Nakamura S, Kondo Y, Matsubae K, Nakajima K, Nagasaka T (2011) UPIOM: a new tool of MFA and its application to the flow of iron and steel associated with car production. Environ Sci Technol 45(3):1114-1120

Östblom G (1993) Increasing foreign supply of intermediates and less reliance on domestic resources: the production structure of the Swedish economy, 1957-1980. Empir Econ 18(3):481-500

Östblom G (1997) Use of the convergence condition for triangularizing input-output matrices and the similarity of production structures among Nordic countries 1970, 1980 and 1985. Struct Chang Econ Dyn 8(1):481-500

Peters GP, Minx JC, Weber CL, Edenhofer O (2011) Growth in emission transfers via international trade from 1990 to 2008. Proc Natl Acad Sci USA 108(21):8903-8908

Pintea CM, Crisan GC, Chira C, Dumitrescu D (2009) A hybrid ant-based approach to the economic triangulation problem for input-output tables. In: Corchado E, Wu X, Oja E, Herrero Á, Baruque B (eds) Hybrid artificial intelligence systems. Lecture notes in computer science, vol 5572. Springer, Berlin, pp 376-383

Pryor FL (1994) Growth deceleration and transaction costs: a note. J Econ Behav Organ 25(1):121-133

SB-MIAC [Statistics Bureau, Ministry of Internal Affairs and Communications, Government of Japan] (2011) 1995-2000-2005 linked input-output table for Japan. http://www.stat.go.jp/data/io/link/ link05.htm. Accessed 10 Sept 2013

Santhanam KW, Patil RH (1972) A study of the production structure of the Indian economy: an international comparison. Econometrica 40(1):159-176

Simpson D, Tsukui J (1965) The fundamental structure of input-output tables: an international comparison. Rev Econ Stat 47(4):434-446 
Song BN (1977) The production structure of the Korean economy: international and historical comparisons. Econometrica 45(1):147-162

Steen-Olsen K, Weinzettel J, Cranston G, Ercin AE, Hertwich EG (2012) Carbon, land, and water footprint accounts for the European Union: consumption, production, and displacements through international trade. Environ Sci Technol 46(20):10883-10891

Timmer MP (ed) (2012) The world input-output database (WIOD): contents, sources and methods. WIOD working paper 10. http://www.wiod.org. Accessed 9 Sept 2013

Tukker A, Dietzenbacher E (2013) Global multiregional input-output frameworks: an introduction and outlook. Econ Syst Res 25(1):1-19

Wiedmann TO, Schandl H, Lenzen M, Moran D, Suh S, West J, Kanemoto K (2013) The material footprint of nations. Proc Natl Acad Sci USA. doi:10.1073/pnas.1220362110 